\title{
Photocarrier Transport Property of Phenylene-vinylene Dendrimer Studied by Time-of-Flight Method
}

\author{
By Masanobu MIZUSAKI, ${ }^{1, *}$ Motohiro YAMAHARA,,${ }^{1, \dagger}$ Yuichiro YAMADA, ${ }^{1}$ Satoru OBARA, ${ }^{2}$ and Kentaro TADA ${ }^{2}$
}

The photocarrier transport property of the three-generation dendrimer, which is composed of phenylene-vinylene as the dendron group and triphenylamine as the surface group G3D3-PV(TPA), was studied by the time of flight (TOF) method using a sandwich-type cell of indium-tin-oxide (ITO)/G3D3-PV(TPA)/Mg-Al. The photocurrent decay shows that hole transport occurs stronger than electron transport, and dispersive transport is observed in the dendrimer film. The mobility at $25^{\circ} \mathrm{C}$ exhibits negative electric field dependence, and the intrinsic mobility at zero electric field is $7.1 \times 10^{-5} \mathrm{~cm}^{2} /(\mathrm{V} \cdot \mathrm{s})$. This is faster than that of linear poly ( $p$-phenylene-vinylene). Besides this, the result is one order smaller than that of poly( 3 hexylthiophene), but faster than those of poly(3-dodecylthiophene) and poly(3-octadecylthiophene). The result indicates that one of the important parameters for the carrier transport is the average distance between $\pi$-conjugated molecules. The fact that the carrier hopping of the G3D3-PV(TPA) dendrimer occurs effectively implies the structure would be a disk shape since the phenylene-vinylene dendron sites of the different dendrimer molecule are estimated to be close to each other.

KEY WORDS: $\pi$-Conjugated Soluble Dendrimer / Carrier Transport / Mobility / Time of Flight /

Recently, $\pi$-conjugated polymer materials have attracted much interest as potential candidates for use in electro-optical devices, since they exhibit unique features such as flexibility, solubility, and easy film preparation with uniform thickness in large area. ${ }^{1-5}$ In particular, $\pi$-conjugated dendrimers are one of the most promising materials because they show multi-functional properties such as electrical- and/or optical-properties, and high solubility with one molecule. ${ }^{6-12}$ The dendrimer consists of a core, dendrons (branches), and surface groups. The core and the dendron define the key electrical- or opticalproperties such as carrier transport or light emission. Besides this, the dendron attached to the core can modify the electricalor optical-properties by adjusting the generation number of the dendrimer. ${ }^{8}$ Here, the generation number of the dendrimer is defined as the number of branching levels from the core, and this can be used to control the size or the shape of the dendrimer. Therefore, the generation number has been shown to give control of the carrier transport or the light emission property. The surface groups are at the distal ends of the dendrimer, and therefore primarily define the solubility and processing properties.

The carrier transport property of the solution-processible $\pi$-conjugated-polymer or the dendrimer has been studied widely. ${ }^{7,13-17}$ It is known that the kinds of solvents, soluble moieties attached to the polymers or the dendrimers, and spinning conditions can produce very different thin-film morphologies. In addition, even in relatively high ordered materials such as ladder-type polymers, sample history has been shown to have a drastic effect on the mobility of the carrier transport or the optical property. ${ }^{18}$ This may partly explain the wide range of mobility measured for some of these materials. ${ }^{19}$ The mobility of the carrier along the polymer chain can be quite high and, in these cases, the rate-limiting step is degree of disorder in the material. ${ }^{1}$ This is usually called "carrier-hopping" model as shown in Figure 1(a). Figure 1(a) illustrates the conceptual model of the polymer chains and the carrier-hopping. The degree of the molecular order level makes the optimization of the mobility for most ladder-type polymer materials. On the contrary, in case of the globular-type materials such as a dendrimer, the $\pi$-conjugated moieties are separated by the surface groups as shown in Figure 1(b). Therefore, it is not easy for the carrier to transport in the dendrimer film. This kind of insight promotes us to study the carrier transport property of the $\pi$-conjugated solution-processible dendrimer, which is attached to the soluble molecule as the surface group. In this study, we focus on the carrier transport property of the dendrimer, which is composed of $\pi$-conjugated molecule phenylene-vinylene as the dendron and of triphenylamine as the surface group G3D3-PV(TPA) shown in Figure 2(a), by the time of flight (TOF) method. In particular, there are no reports related to the carrier transport property of the $\pi$-conjugated dendrimer covered by the bulky surface group.

\section{EXPERIMENTAL}

\section{Materials}

The chemical structure of the dendrimer, G3D3-PV(TPA), used in this study is shown in Figure 2(a). The synthesis procedure was reported previously. ${ }^{20}$ The core and the dendron are constructed from phenylene-vinylene, which shows the carrier transport property. In the following of this paper, we determine both the core and the dendron as the "dendron"

\footnotetext{
${ }^{1}$ Corporate Research and Development Group, Sharp Corp., 2613-1 Ichinomoto-cho, Tenri 632-8567, Japan

2Photosensitive Materials Research Center, Toyo Gosei Co. Ltd., 4-2-1 Wakahagi, Inba-mura, Inba-gun 270-1609, Japan

'Present Affiliation: SUMITOMO CHEMICAL Co. Ltd., IT-Related Chemicals Research Laboratory

*To whom correspondence should be addressed (Tel: +81-743-65-3876, Fax: +81-743-65-4045, E-mail: mizusaki.masanobu @ sharp.co.jp).
} 
(a) Ladder-Type Polymer

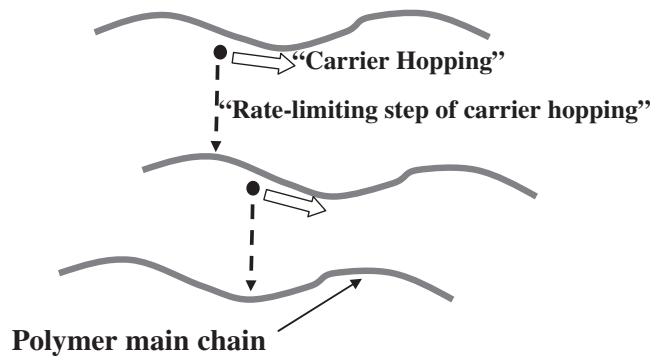

(b) Dendrimer

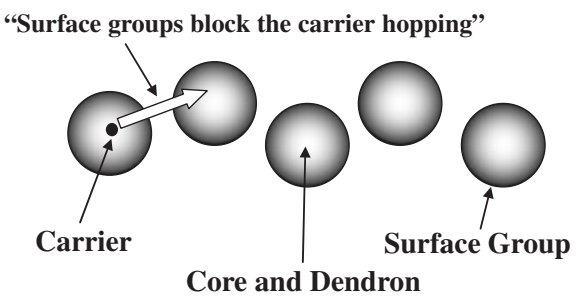

Figure 1. Conceptual illustrations of the carrier transport for (a) ladder-type polymer and (b) dendrimer.

because both are constructed from the same molecule. The surface group is constructed from a bulky moiety triphenylamine. The weight and number average molecular weights measured by GPC are 7,700 and 7,200, respectively, and the molecular weight distribution is $1.072 .^{20}$ The solution of the G3D3-PV(TPA) dendrimer was prepared in chloroform at concentration of typically $10 \mathrm{mg} / \mathrm{mL}$. Indium tin oxide (ITO) substrates were masked, patterned and cleaned in ultrasonic of $\mathrm{NaOH}$ and deionized water. The deionized water used for cleaning of the ITO substrate was from the Millipore water purification system. Films of G3D3-PV(TPA), typically $1 \mu \mathrm{m}$ thick, were then formed by spin-coating from the chloroform solutions onto the cleaned ITO substrates at $25^{\circ} \mathrm{C}$. A vacuum evaporator was used for the thermal evaporation of the alloy of $10 \mathrm{wt} \% \mathrm{Mg}$ and $90 \mathrm{wt} \% \mathrm{Al}(\mathrm{Mg}-\mathrm{Al})$ onto the desired ITO glass plates deposited with the $1 \mu \mathrm{m}$ dendrimer film.

Another dendrimer, G2D3-BE(TPB-TPA) shown in Figure 2(b), was also prepared in order to evaluate the carrier transport property of the triphenylamine surface group. The G2D3-BE(TPB-TPA) dendrimer consists of benzyl-ether dendron, which is not shown the carrier transport property, and the triphenylamine surface group. The weight and number average molecular weights measured by GPC are 5,000 and 4,800, respectively, and the molecular weight distribution is 1.042 . The method for sample preparation for the TOF measurement was the same described above.

\section{Mobility}

TOF measurements for ITO/G3D3-PV(TPA)/Mg-Al and ITO/G2D3-BE(TPB-TPA)/Mg-Al cells were carried out with a conventional setup, as described by Chen et al. ${ }^{15}$ A pulsed $\mathrm{N}_{2}$

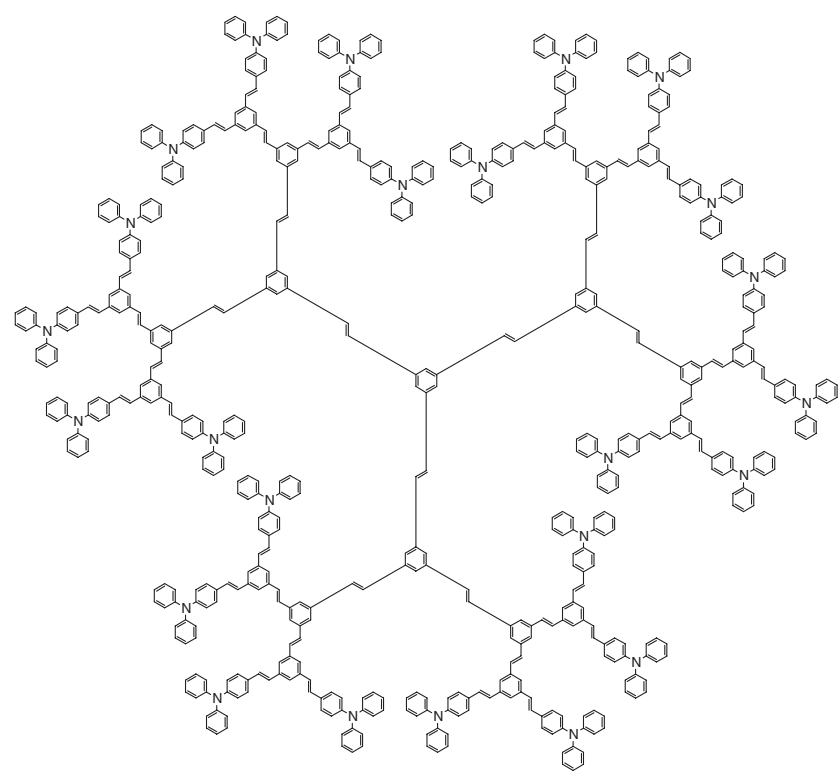

(a)

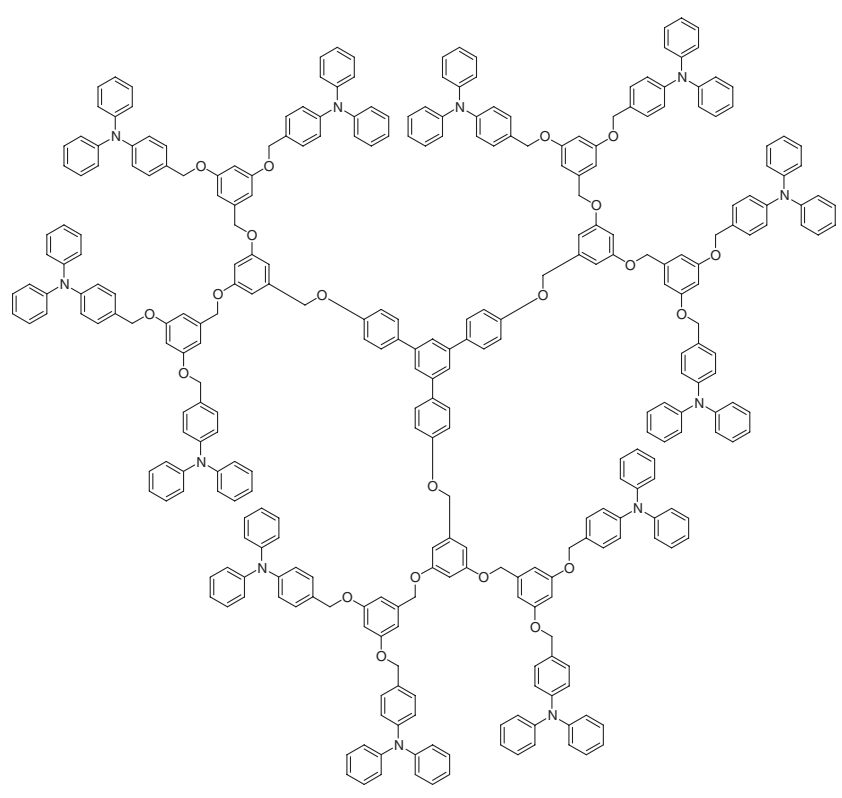

(b)

Figure 2. (a) G3D3-PV(TPA) dendrimer used in this study. (b) G2D3BE(TPB-TPA) dendrimer used for comparison.

laser (337.1 nm, pulse width $0.7 \mathrm{~ns}$ ) having an output of $150 \mu \mathrm{J} /$ pulse was used to create the photocarriers in the transparent ITO electrode comprising the ITO/G3D3-PV(TPA)/Mg-Al or the ITO/G2D3-BE(TPB-TPA)/Mg-Al cell. The measurements were performed at $25^{\circ} \mathrm{C}$ in vacuum condition $\left(10^{-3}\right.$ Torr). The current generated as a result of photocarrier drift was collected across a $50 \Omega$ resister upon the application of an external bias voltage for about $50-100 \mathrm{~ms}$.

\section{RESULTS AND DISCUSSION}

For carrier generation by the irradiation of $\mathrm{N}_{2}$ laser, it is 


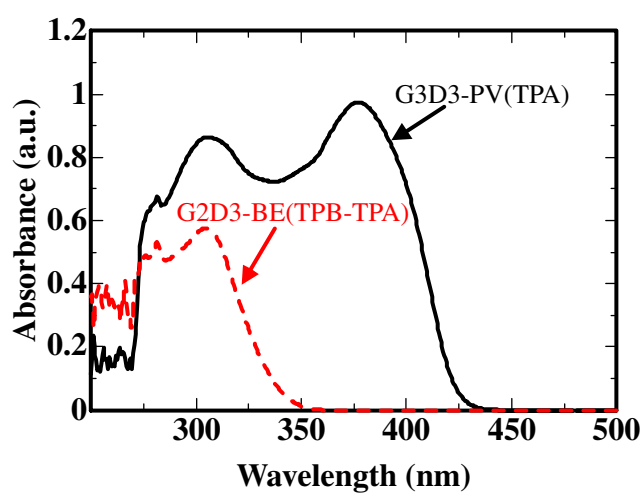

Figure 3. UV spectra for G3D3-PV(TPA) and G2D3-BE(TPB-TPA) dendrimers.

indispensable to show the absorption at $337.1 \mathrm{~nm}$. The UV spectra for G3D3-PV(TPA) and G2D3-BE(TPB-TPA) dendrimer films are shown in Figure 3. The absorption centered at $300 \mathrm{~nm}$ observed both the dendrimers is from the triphenylamine group. On the other hand, the absorption centered at $370 \mathrm{~nm}$ observed the G3D3-PV(TPA) dendrimer is from the phenylene-vinylene dendron. Since both the dendrimers show the absorption at $337.1 \mathrm{~nm}$, the photoexcited carriers generate at the dendrimer layers.

For TOF measurements, the $\mathrm{N}_{2}$ laser light was illuminated from ITO side and the photoexcited carriers of hole and electron generate at the surface of the dendrimer films based on Beer's law. In the case that positive bias voltage is applied at the ITO side, the hole drifts from the ITO side to the Mg-Al side. On the other hand, the electron drifts as the negative bias voltage is applied at the ITO side. Those are depicted in Figure 4(a) and 4(b), respectively. Typical transient photocurrents at applied voltage of $+50 \mathrm{~V}$ and $-50 \mathrm{~V}$ are shown in Figure 5(a) and 5(b), respectively. In case of the G3D3-

(a)

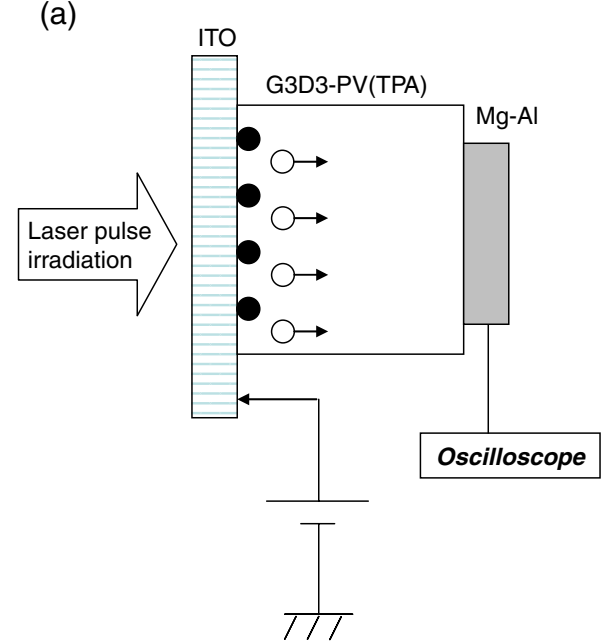

(a)

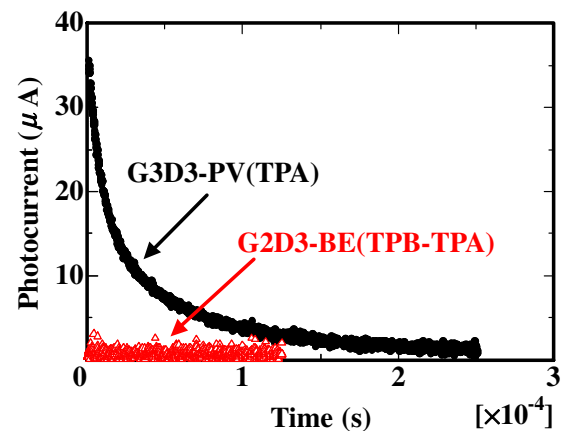

(b)

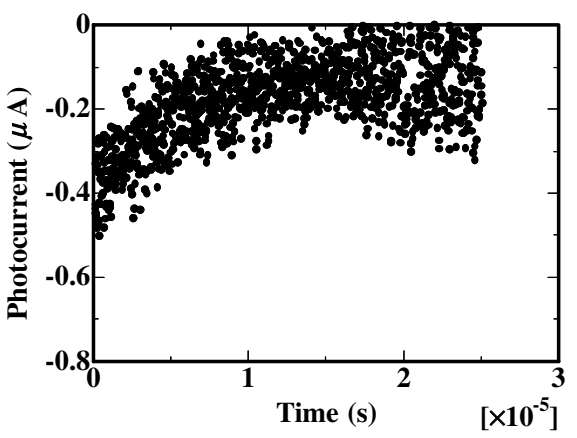

Figure 5. Profiles of transient photocurrent for ITO/G3P3-PV(TPA)/Mg-Al and ITO/G2P3-BE(TPB-TPA)/Mg-Al cells; transient photocuurent from (a) hole transport, and (b) electron transport.

PV(TPA) dendrimer, when the positive bias voltage is applied at the ITO electrode against the $\mathrm{Mg}$-Al electrode, much larger photocurrent is observed compared with the case that the negative bias voltage is applied at the ITO side. This is indicative that the hole transport occurs preferentially compared with the electron transport. On the other hand, in case of the G2D3-BE(TPB-TPA) dendrimer, the photocurrent was not observed when the $+50 \mathrm{~V}$ was applied to the cell. This implies

(b)

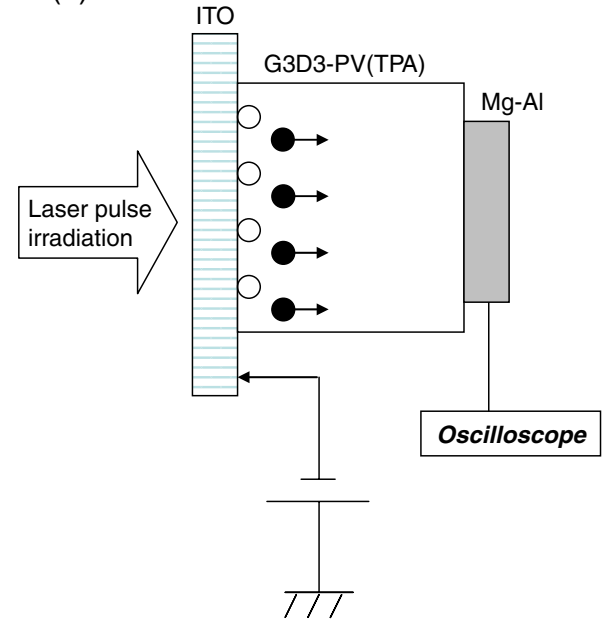

Hole

Electron

Figure 4. Schematic illustrations of TOF measurement system; (a) positive bias is applied at ITO side, and (b) negative bias is applied at ITO side. 


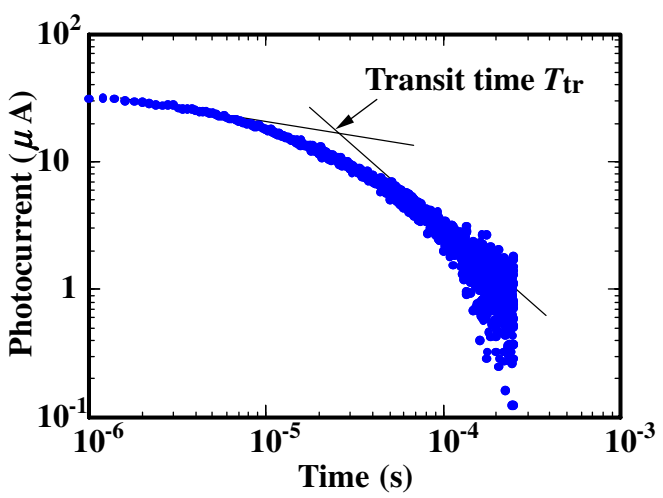

Figure 6. Double logarithmic plot of the positive transient photocurrent for ITO/G3D3-PV(TPA)/Mg-Al cell.

that the hole transport does not occur in the triphenylamine surface group prepared by spin-coating from the solutions. According to Figure 5(a), the profile of the positive transient photocurrent for the G3D3-PV(TPA) dendrimer is typically dispersive. This result is same as the results of $\operatorname{poly}(p$ phenylene-vinylene) and poly(3-hexylthiophene), indicating that the carrier hopping property in the dendrimer film is same as that in the ladder type polymer film. ${ }^{13,21}$ This result is also same as the result of the dendrimer film without bulky surface group reported by Lupton et al., implying that the phenylenevinylene dendron sites between the different dendrimer molecule are close to each other even the dendron sites are surrounded by the bulky surface group. ${ }^{9}$

Figure 6 shows the double logarithmic plot (Scher-Montroll plot) of the transient photocurrent of the hole for the ITO/ G3D3-PV(TPA)/Mg-Al cell at $25^{\circ} \mathrm{C}$, which is modified of Figure 5(a). The time estimated from the intercept of two tangential lines before and after the inflection point of the photocurrent as shown in Figure 6 is termed the transit time $T_{\mathrm{tr}}{ }^{22} T_{\mathrm{tr}}$ is defined as the time required for the photocarrier to travel across the film thickness $L .^{22}$ It is known that the relationship between $T_{\text {tr }}$ and $L$ is as follows;

$$
T_{\mathrm{tr}}=\frac{L}{\mu E}
$$

where $E$ is the electric field and indicated as $V / L$, which $V$ is the applied bias voltage, and $\mu$ is the constant known as the carrier mobility. Therefore, eq (1) is modified as follows.

$$
T_{\mathrm{tr}}=\frac{L^{2}}{\mu V}
$$

According to eq (2), $T_{\text {tr }}$ becomes inversely proportinal to $V$. Figure 7 shows $T_{\text {tr }}$ as a function of $V . T_{\text {tr }}$ is reversely proportional to $V$. However, $T_{\text {tr }}$ does not fit well with the theoretical result which is derived from eq (2). On the other hand, we also plotted the current at the transit time $I_{\text {tr }}$ as a function of $V$ in Figure 8. $I_{\text {tr }}$ increases linearly with increasing $V$, implying that the number of the transporting carrier increases linearly with increasing $V$. Based on the results of Figures 7 and 8 , we estimated that $\mu$ changes with $V$. Figure 9

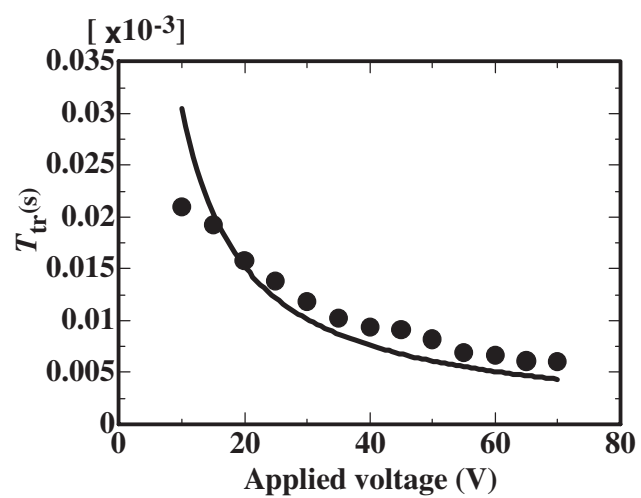

Figure 7. Transit time $T_{\mathrm{tr}}$ as a function of $V$.

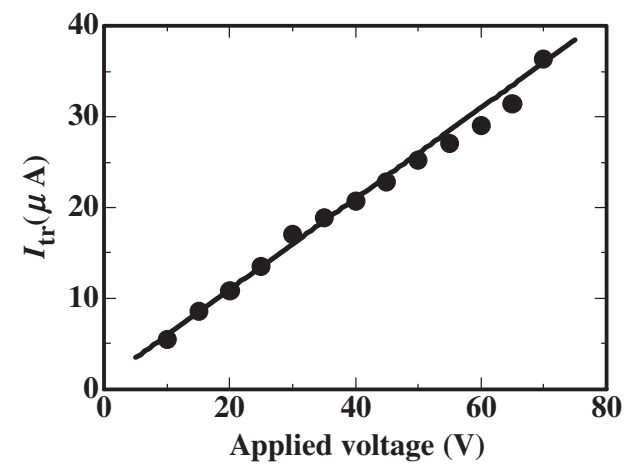

Figure 8. Current at the transit time $I_{\mathrm{tr}}$ as a function of $V$.

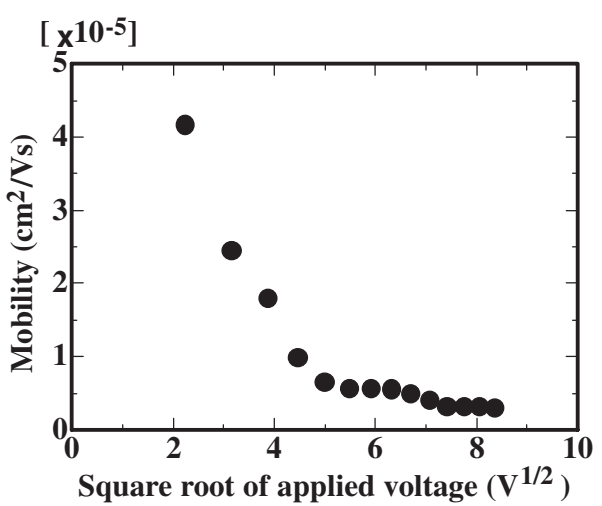

Figure 9. Mobility $\mu$ as a function of $V^{1 / 2}$.

shows $\mu$ as a function of the square root of the applied bias voltage $V^{1 / 2}$. The mobility $\mu$ decreases with increasing $V^{1 / 2}$, indicating the possibility that the nonuniform electric field is applied to the G3D3-PV(TPA) dendrimer film as described by Kaneto et al. ${ }^{22,23}$ They proposed a model of heterogeneous electric field distribution for the cell with the Schottky junction at the surface of the $\mathrm{Mg}$-Al electrode as depicted in Figure 10. The transit time $T_{\text {tr }}$ for the photocarrier to the opposite surface is determined as follows: ${ }^{22,23}$

$$
T_{\mathrm{tr}}=T_{\mathrm{d}}+T_{\mathrm{b}}
$$




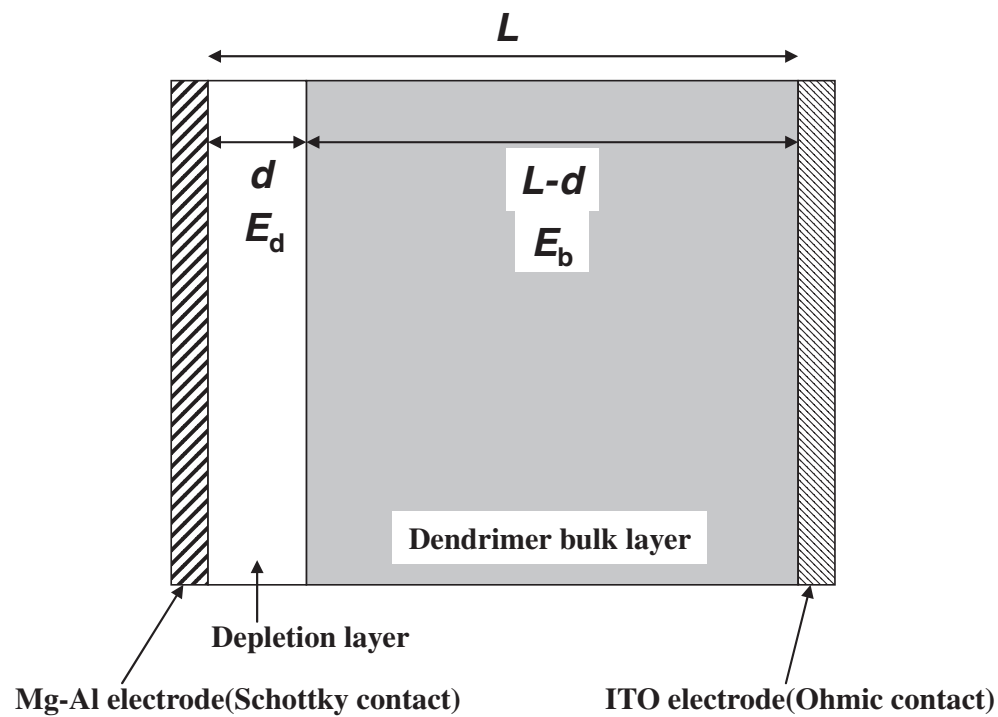

Figure 10. A model of double electric field distribution for Schottky junction of electrode and G3D3-PV(TPA) layer.

where $T_{\mathrm{d}}$ and $T_{\mathrm{b}}$ are the transit times of depletion layer and bulk layer, respectively. Those are determined in the followings:

$$
\begin{aligned}
& T_{\mathrm{d}}=\frac{d}{\mu E_{\mathrm{d}}}=\frac{\sigma_{\mathrm{d}}(L-d)+\sigma_{\mathrm{b}} d}{\mu \sigma_{\mathrm{b}} d V} d^{2} \\
& T_{\mathrm{b}}=\frac{L-d}{\mu E_{\mathrm{b}}}=\frac{\sigma_{\mathrm{d}}(L-d)+\sigma_{\mathrm{b}} d}{\mu \sigma_{\mathrm{d}}(L-d) V}(L-d)^{2}
\end{aligned}
$$

where $E_{\mathrm{d}}$ and $E_{\mathrm{b}}$ are the electric fields for the depletion layer and the bulk layer, respectively, $\sigma_{\mathrm{d}}$ and $\sigma_{\mathrm{b}}$ are the conductivity of the depletion layer and the bulk layer, respectively, $d$ is the thickness of the depletion layer, and $L$ is the thickness of the dendrimer film. If it is assumed that $L \gg d$ and $\sigma_{\mathrm{d}} \ll \sigma_{\mathrm{b}}$, the following relation is obtained. ${ }^{22,23}$

$$
T_{\mathrm{tr}} V \approx \frac{L^{2}}{\mu}\left(1+\frac{\sigma_{b}}{\sigma_{d} L} d\right)
$$

According to eq (4), $d$ is proportional to $V^{1 / 2}$. Then, eq (6) indicates that the product of $T_{\text {tr }}$ and $V\left(T_{\text {tr }} \cdot V\right)$ is proportional to $V^{1 / 2}$. Figure 11 shows the plot for $T_{\text {tr }} \cdot V$ as a function of $V^{1 / 2}$, showing the linear relation. The result indicates that the double layer model which is composed of the bulk layer and of the depletion layer is valid in our cell. The intercept at $V^{1 / 2} \rightarrow$ 0 is $1.4 \times 10^{-4}(\mathrm{~V} \cdot \mathrm{s})$ from the extrapolation of the plot, hence the mobility obtained is $7.1 \times 10^{-5} \mathrm{~cm}^{2} /(\mathrm{V} \cdot \mathrm{s})$ since the thickness of the G3D3-PV(TPA) dendrimer film is $1 \mu \mathrm{m}$ $\left(1 \times 10^{-4} \mathrm{~cm}\right)$.

The hole mobility for linear polymer, $\operatorname{poly}(p$-phenylenevinylene), measured by the TOF method was reported to be in the range of $10^{-5} \mathrm{~cm}^{2} /(\mathrm{V} \cdot \mathrm{s})$ at room temperature. ${ }^{21}$ The mobility is the same order as that of the G3D3-PV(TPA) dendrimer though the dendrimer is surrounded by the triphenylamine surface group. The fact implies that the phenylenevinylene dendron sites of the different dendrimer molecule are close to each other.

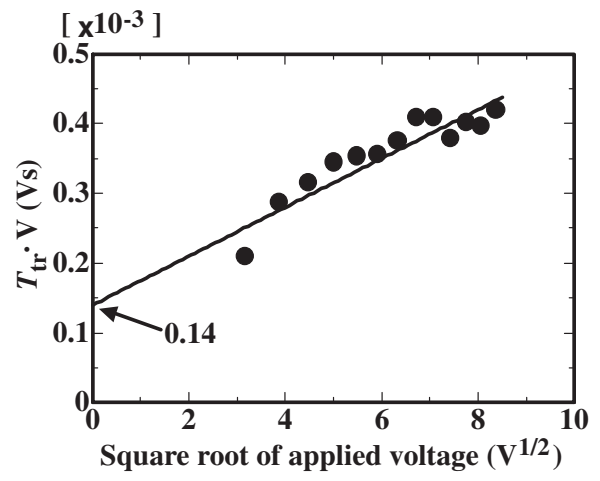

Figure 11. Product of $T_{\mathrm{tr}}$ and $V\left(T_{\mathrm{tr}} \cdot V\right)$ as a function of $V^{1 / 2}$.

Besides this, Pandey et al. reported that the mobility for poly(3-hexylthiophene) measured by the TOF method was $10^{-4} \mathrm{~cm}^{2} /(\mathrm{V} \cdot \mathrm{s})$ order. ${ }^{24}$ They also reported that those for poly(3-dodecylthiophene) and poly(3-octadecylthiophene) were $8 \times 10^{-6} \mathrm{~cm}^{2} /(\mathrm{V} \cdot \mathrm{s})$ and $3 \times 10^{-6} \mathrm{~cm}^{2} /(\mathrm{V} \cdot \mathrm{s})$, respectively. ${ }^{24}$ The mobility of the G3D3-PV(TPA) dendrimer is slower than that of poly(3-hexylthiophene), but faster than those of poly(3-dodecylthiophene) and poly(3-octadecylthiophene). We estimate that one of the important parameters for the carrier transport is the average distance between $\pi$-conjugated molecules. The carrier hopping rate is estimated to decrease with increasing the average distance between the $\pi$-conjugated molecules. If we assume that the structure of the G3D3PV(TPA) dendrimer is a globular shape, the $\pi$-conjugated sites of phenylene-vinylene with different dendrimer molecule are perfectly separated by the surface group of triphenylamine. Then, the carrier hopping rate is strictly limited. However, the facts that the mobility of the G3D3-PV(TPA) dendrimer is the same order as that of poly ( $p$-phenylene-vinylene) and is faster than those of poly(3-dodecylthiophene) and poly(3-octadec- 
ylthiophene) indicate that the phenylene-vinylene molecules with different dendrimer molecule are comparably close to each other. Therefore, the structure of the dendrimer we used in this study, G3D3-PV(TPA), is not the globular shape but the disk shape.

\section{CONCLUSIONS}

We clarified the carrier transport property of the G3D3PV(TPA) dendrimer having bulky surface group by the TOF method using the ITO/G3D3-PV(TPA)/Mg-Al cell. The transport property of the hole was strongly shown compared with that of the electron, and dispersive transport was observed in the dendrimer film. The mobility at $25^{\circ} \mathrm{C}$ was decreased with increasing the electric field, indicating the nonumiform electric field distribution due to the formation of the depletion layer in the dendrimer film is formed. The intrinsic mobility at zero electric field was $7.1 \times 10^{-5} \mathrm{~cm}^{2} /(\mathrm{V} \cdot \mathrm{s})$. This is the same order as that of poly ( $p$-phenylene-vinylene) though poly $(p$ phenylene-vinylene) has not the soluble triphenylamine group. ${ }^{21}$ Besides this, the mobility for the G3D3-PV(TPA) dendrimer is one order smaller than that of poly(3-hexylthiophene), but faster than those of poly(3-dodecylthiophene) and poly(3-octadecylthiophene). ${ }^{24}$ Those comparisons are indicative that the carrier transport occurs effectively with decreasing the average distance between the $\pi$-conjugated molecules. The structure of the G3D3-PV(TPA) dendrimer is the disk shape since the dendron sites of phenylene-vinylene with different dendrimer molecule are close to each other. The important parameter for the molecular architecture of the soluble carrier transport dendrimer is that the structure would be the disk shape.

Received: September 22, 2008

Accepted: November 20, 2008

Published: January 7, 2009

\section{REFERENCES}

1. R. J. O. M. Hoofman, M. P. de Haas, L. D. A. Siebbeles, and J. M. Warman, Nature, 392, 54 (1998).
2. M. Redecker, D. D. C. Bradley, M. Inbasekaran, and E. P. Woo, Appl. Phys. Lett., 73, 1565 (1998).

3. T.-A. Chen, X. Wu, and R. D. Rieke, J. Am. Chem. Soc., 117, 233 (1995).

4. K. Kaneto, S. Hayashi, S. Ura, and K. Yoshini, J. Phys. Soc. Jpn., 54, 1146 (1985).

5. S. Annapoorni, N. S. Sudaresan, S. S. Pandey, and B. D. Malhotra, J. Appl. Phys., 74, 2109 (1993).

6. T. D. Anthopoulos, J. P. J. Markham, E. B. Namdas, J. R. Lawrence, I. D. W. Samuel, S.-C. Lo, and P. L. Burn, Org. Elec., 4, 71 (2003).

7. J. P. J. Markham, I. D. W. Samuel, S.-C. Lo, P. L. Burn, M. Weiter, and H. Bassler, J. Appl. Phys., 95, 438 (2004).

8. J. M. Lupton, I. D. W. Samuel, R. Beavington, M. J. Frampton, P. L. Burn, and H. Bassler, Phys. Rev. B., 63, 155206 (2001).

9. J. M. Lupton, I. D. W. Samuel, R. Beavington, P. L. Burn, and H. Bassler, Synth. Met., 121, 1703 (2001).

10. J. N. G. Pillow, M. Halim, J. M. Lupton, P. L. Burn, and I. D. W. Samuel, Macromolecules, 32, 5985 (1999).

11. S.-B. Jung, S.-Y. Yoo, E. Park, C. Kim, and Y.-S. Kwon, Jpn. J. Appl. Phys., Part 1, 41, 3065 (2002).

12. S.-B. Jung, S.-Y. Yoo, C. Kim, and Y.-S. Kwon, Jpn. J. Appl. Phys., Part 1, 42, 2434 (2003).

13. S. S. Pandey, W. Takashima, S. Nagamatsu, and K. Kaneto, IEICE Trans. Elec., E83-C, 1088 (2000).

14. K. Kaneto, K. Hatae, S. Nagamatsu, W. Takashima, S. S. Pandey, K. Endo, and M. Rikukawa, Jpn. J. Appl. Phys., Part 2, 38, L1188 (1999).

15. B. Chen, C.-S. Lee, S.-T. Lee, P. Webb, Y.-C. Chan, W. Gambling, H. Tian, and W. Zhu, Jpn. J. Appl. Phys., Part 2, 39, 1190 (2000).

16. B. D. Malhotra, W. Takashima, S. S. Pandey, R. Singhal, K. Endo, M. Rikukawa, and K. Kaneto, Jpn. J. Appl. Phys., Part 2, 38, 6768 (1999).

17. D. Poplavskyy and J. Nelson, J. Appl. Phys., 93, 341 (2003).

18. D. Hertel, H. Bassler, U. Scherf, and H. H. Horhold, J. Chem. Phys., 110, 9214 (1999)

19. H. C. F. Martens, P. W. M. Blom, and H. F. M. Schoo, Phys. Rev. B., 61, 7489 (2000).

20. M. Yamahara, S. Obara, and K. Tada, Jpn. Kokai, TOKKYO Koho, No. 2004-18539 (2004).

21. E. Lebedev, Th. Dittrich, V. Petrova-Koch, S. Karg, and W. Brutting, Appl. Phys. Lett., 71, 2686 (1997).

22. K. Kaneto, S. S. Pandey, and W. Takashima, Jpn. J. Appl. Phys., Part 1, 40, 4933 (2001).

23. K. Kaneto, K. Takayama, W. Takashima, T. Endo, and M. Rikukawa, Jpn. J. Appl. Phys., Part 1, 41, 675 (2002).

24. S. S. Pandey, S. Nagamatsu, W. Takashima, and K. Kaneto, Jpn. J. Appl. Phys., Part 1, 39, 6309 (2000). 